\title{
Isolated Gross Hematuria or Associated to Acute Retention of Urine as a Sign of Urologic Malignant Pathology
}

\author{
Angel Tomas Ibáñez Concejo, Marco Antonio Castillo, Juan Sanchez-Verde Bilbao, Jorge Short Apellaniz, \\ Ambar Deschamps Perdomo and Joaquin Garcia Cañete \\ Emergency Department, Fundación Jiménez-Díaz Hospital, Madrid 28760, Spain
}

Received: December 30, 2013 / Accepted: January 16, 2014 / Published: January 31, 2014.

\begin{abstract}
The causes of IGH (Isolated gross hematuria), as the only symptom or associated with ARU (acute retention of urine) caused by clots in the urinary tract in patients who do not have a previous diagnosis of urological malignant pathology and to study the immediate mortality in patients with that sign are described. A descriptive observational study of the etiologies of patients shows the above mentioned symptomatology from the 1st of January to the 30th of June of 2008 in the Emergency Department which received follow-up treatment up to December 2010. It also describes the mortality before the definitive diagnosis and the days between receiving attention in the Emergencies Department and the diagnostic tests. One hundred and four cases with the criteria described above were evaluated. Of these, $20.0 \%$ turned out to have bladder, prostate or kidney cancer; the bladder being the most affected organ. Of all the pathology, benign and malignant, the most frequent one is the benign hypertrophy of the prostate (49.8\% of the total). Of the total, $4.8 \%$ of the patients died in the following weeks before finishing the diagnostic study. Isolated gross hematuria is a clinical symptom closely related to urological malignant pathology and it does not have an insignificant mortality rate in a short period of time. For these reasons it is necessary to accelerate the diagnostic tests as soon as the patient presents the symptom.
\end{abstract}

Key words: Gross hematuria, bladder cancer, prostate cancer, benign hypertrophy of the prostate, urothelioma.

\section{Introduction}

Gross hematuria is defined as red-coloured urine or the presence of more than 100 red cells per field in the urine sediment, being a common cause of treatment in the Emergency Department. Hematuria means there is bleeding in the urinary tract, upper or lower.

Gross hematuria can appear as a clinical sign within a syndrome, appearing in the context of an acute pain in the renal flank or fossa as in acute renal cholic; and it can be associated with fever, dysuria and urinary frequency in a urinary infection or a trauma in the renal flank. The hematuria which is more frequently associated with malignant pathology is on the other hand more silent and painless and only when the

Corresponding author: Angel Tomas Ibáñez Concejo, M.D., Ph.D., research fields: emergency medicine and urology. E-mail: tomasibanezconcejo@msn.com. hematuria is very clear might it entail the presence of clots that block the urinary tract and create intense pain.

There were several studies that dealt with the etiologic cause of hematuria, but only a few of them detailed in what percentage of these patients who had already a diagnosis of urological malignant pathology. Clinicians on their everyday practice know that many patients consult after a prostate or bladder surgery or after undergoing an aggressive treatment because they are still bleeding or are patients already diagnosed of cancer and this is progressing or recurring. These groups of patients have a known malignant cause of hematuria and have had diagnostic protocol carried out, and they should be distinguished from recent cases of hematuria, in which the cause is unknown and could be benign or malignant. Why do the authors need to make this distinction? Well because failing to do so might 
lead to an overestimation of neoplasic causes and what is really important is to know the probability for a hematuria to be finally secondary to a malignant process.

Another important issue is BPH (benign porstatic hyperplasia) a very prevalent pathology in males over 50 years that in some occasions presents with gross hematuria. One might consider that patients with BPH should not be included as cases of undiagnosed hematuria, but due to its high prevalence, every patient with this diagnosis should undergo the same diagnostic tests as any patient is presenting a first episode of gross hematuria, therefore they have not been excluded from the study.

Finally, it is true that gross hematuria is rarely so great as to generate haemodynamic instability of the patient but it can also hide a severe pathology that has to be diagnosed and treated early and appropriately. Given its relation to cancer and it is not insignificant mortality rate, as will be seen in the study, gross hematuria should be dealt with in a protocolised manner as a warning symptom.

\section{Methods and Materials}

A descriptive study was done of IGH (isolated gross hematuria) or ARU (acute retention of urine) cases seen during the first 6 months of 2008 in the Emergency Department of the authors' hospital. Hematuria in other clinical scenario, such as a trauma, renal colic or urinary track infection, was excluded.

The sample was selected from the medical-surgery area of the Emergencies Department between the 1st of January and the 30th of June, 2008. Pediatric, gynecological or of talmological emergencies which in the hospital are dealt with in other departments were excluded. This gives a total of 28,173 cases, of which 153 had a diagnosis of IGH (133) or AUR (20).

All those already diagnosed with a urological malignant pathology were excluded (49 cases), given a total of 104 cases included in the study.

Search made on hospitals records, INDRA ${ }^{\circledR}$ data base, for the final diagnosis was used to complete the necessary information.

The variables that were studied were: age, sex, smoking habits at any stage in life, time lapsed between their episode in the urgency department and the exact date of the diagnosis, the final diagnosis and in the case of malignant pathology necessary treatment and mortality.

\section{Results}

Overall gross hematuria represents $0.54 \%$ of all visits to the emergency department, 104 patients met the study selection criteria. The average age at presentation was 70 years with a median of 77 years, $86.5 \%$ of cases presented on man (Table 1). Within the malignant pathology group, the youngest patient was 61 years old and in the benign group there were 13 patients under that age (the most common pathology was the BPH with 6 cases).

96.16\% (100 patients) of the cases were derived to external urology consultations and $3.84 \%$ (four patients) were hospitalized for an inmediate study. Two patients did not turn up for their urology appointments and five of them died before finishing the study.

Of the patients who died, two of them died when they were hospitalized in the urology department days after arriving at the Emergency Department with the diagnosis of hipovolemic shock and urinary sepsis. And three patients who were not hospitalized initially died of another cause, two in the hospital with the diagnosis of ischaemic stroke and congestive heart failure, and the other patient in another hospital with the diagnosis of heart failure.

In $21(20.19 \%)$ cases the final diagnosis was a malignant neoplasia, with bladder cancer (15 cases) followed by prostate adenocarcinoma (three cases) and

Table 1 Sex description.

\begin{tabular}{lrr}
\hline Sex & N & $\%$ \\
\hline Males & 90 & 86.5 \\
Females & 14 & 13.5 \\
Total & 104 & 100.0 \\
\hline
\end{tabular}


kidney neoplasia (three cases) and only one case of uretral urotelioma (Table 2).

Of the 15 bladder cases 14 were urotelioma and one carcinosarcoma. Of the three kidney cases, two of them were clear cell carcinoma and one of them a renal pelvis urotelioma. The staging is described in Table 3.

In 20 (95\%) of the neoplasias cases were described in males. 15 (71.4\%) of them with a malignant disease, and a $52 \%$ of the patients with BHP or ARU did smoke or had smoked at some stage in their lives (Table 4).

To measure the time elapsed since the patients were discharged from the Emergency Department to the date, the final diagnosis was established, and the median delay was used and turned out to be 140 days; in the case of a malignant pathology the number decreased to a median of 71 days (Table 5).

In $73.08 \%$ of the cases there was no malignant pathology, the most common being BHP, infection of the urinary tract, nonspecific hematuria, urolithiasis, and benign bladder pathology. BHP represents $49.80 \%$ of the total of benign and malignant pathology.

The rest correspond to the patients who died (4.8\%) and $2 \%$ whose cases could not be followed up.

As far mortality data, five patients died before completing the study, corresponding to $4.8 \%$ of the hematuria cases. Additionally, $15 \%$ of the patients that were diagnosed with malignant pathology (three cases) died in the first year after the final diagnosis.

Table 2 Final diagnosis.

\begin{tabular}{lrr}
\hline Final diagnosis & N & $\%$ \\
\hline Bladder cancer & 15 & 14.42 \\
Kidney cancer & 3 & 2.88 \\
Prostate cancer & 3 & 2.88 \\
Benign prostatic hyperplasia & 51 & 49.03 \\
Died before finishing the study. & 5 & 4.80 \\
Hematuria due to oral anticoagulation & 1 & 0.96 \\
Benign bladder pathology & 5 & 4.80 \\
Estenosis uretral & 1 & 0.96 \\
Infección tracto urinario & 8 & 7.69 \\
Litiasis & 3 & 2.88 \\
Trauma & 1 & 0.96 \\
Erectile dysfuction & 1 & 0.96 \\
Nonspecific hematuria/Not followed & 7 & 6.73 \\
Total & 104 & 100 \\
\hline
\end{tabular}

Regarding treatment of patients with malignant pathology (Table 6), of 21 patients, 19 received surgery, some of them with coadjutant treatment that was not revised, and one patient received quimiotherapy and one patient radiotherapy.

\section{Discussion}

Clinical guidelines indicate that hematuria must be investigated in all cases [1] except for the so called benign hematuria in which these can be found

Table 3 Bladder cancer who staging.

\begin{tabular}{lrc}
\hline Bladder Cancer Stage & N & $\%$ \\
\hline STAGE I & 3 & 21.4 \\
STAGE II & 4 & 28.6 \\
STAGE III & 3 & 21.4 \\
STAGE IV & 4 & 28.6 \\
Total & 14 & 100 \\
\hline
\end{tabular}

Table 4 Smokers description.

\begin{tabular}{lrc}
\hline & $\mathrm{N}$ & $\%$ \\
\hline Smokers & 55 & 52.9 \\
No smokers & 38 & 36.5 \\
Unknown & 11 & 10.6 \\
Total & 104 & 100 \\
\hline \multicolumn{3}{l}{ Smokers in malignancy pathology group } \\
\hline Smokers & $\mathrm{N}$ & $\%$ \\
No smokers & 15 & 71.4 \\
Unknown & 4 & 19 \\
Total & 2 & 9.5 \\
\hline
\end{tabular}

Table 5 Time passed since the patients were discharged from the emergency department to the date the final diagnosis.

\begin{tabular}{lc}
\hline Percentile, minimum and maximum & Number of days \\
\hline Mediana & 140.00 \\
25 percentile & 48.00 \\
75 percentile & 210.00 \\
Minium & 3.00 \\
Maximum & 814 \\
\hline
\end{tabular}

Table 6 Definitive treatment in malignancy pathology group.

\begin{tabular}{lrc}
\hline & $\mathrm{N}$ & $\%$ \\
\hline Only chemoterapy & 1 & 4.76 \\
Surgery & 19 & 90.48 \\
Only radiotherapy & 1 & 4.76 \\
Total & 21 & 100 \\
\hline
\end{tabular}


menstruation, intense physical exercise, a viral process or a trauma [2-4] and even in these cases observation is required [5]. The main reason for such a detailed study is the high relation between hematuria and urological cancer, even in microhematuria, although is at least four times less frequent (compared to gross hematuria). Gross hematuria is the initial symptom in $85 \%$ of bladder cancer cases and in 40\% of renal cancer [6].

According to several studies finding malignancy after a first episode of gross hematuria varies from 9\% to $44 \%$ [7], with a big variety in the methodology used [8]; even in the studies of microhematuria the discovery of malignancy varies from $2.5 \%$ to $13 \%$ [1, $6]$. In the study, malignancy was observed in $20 \%$ of the cases, excluding patients that already had a prior diagnosis of neoplasia. If they had been included, the total would have risen to $45 \%$ of the cases.

Bladder cancer is the principal cause of malignancy in the study as in other studies, way ahead of prostate and kidney cancer [3]. As for the kidney tumors, even though the three classic signs of kidney cancer are: hematuria, pain or the appearence of an abdominal mass, they only appeared in $9 \%-16 \%$ of cases, with isolated hematuria [9] appearing on many occasions. So even though thinking about bladder cancer, the most prevalent, talking about hematuria and malignant pathology, kidney cancer should be taken into account, because it is being more aggresive in its evolution.

There are risk factors for malignancy that require a more aggressive study in patients with hematuria: smoking habits, occupational exposure to chemical agents (benzene and aromatic amines), age over 40, recidivant urine infections, a clinical history of analgesic abuse, pelvic irradiation in the past or previous gross hematuria $[1,4,10]$. As regarding age, some studies do decrease the age to 35 [4, 9]. Gross hematuria on its own is already a risk factor, although it will be more probable if other risk factors are added. Only a few authors say that gross hematuria can be considered benign in women under 40 or when there are clinical signs of urine infection and a positive urine culture [11]. Of all the risk factors the authors have only described smoking habit, finding a higher prevalence of patients with smoking habits in the group of malignancy as previous studies do.

Fortunately, the greatest percentage of hematuria corresponded to $\mathrm{BPH}$, which was secondary to the vascular congestion in the prostate gland and delated veins in the bladder neck [9]. In previous studies this percentage ranged from $13.5 \%$ to $25 \%$ [12, 13], but it is higher in the study (48\%), it may be due to the fact that other studies did not distinguish between microhemturia and gross hematuria and that in the study patients with previous diagnosis of tumoral pathology were excluded, which underestimated the total, and was not the case for patients who had BHP for reasons set out earlier.

In the authors' results, it might exists an underestimation of gross hematuria as the presenting sing of renal disease, blood dyscrasia or metabolic alteration, although pediatric age had been intentionally excluded from the study, since these pathologies often showed microhematuria [9]. In previous studies of gross hematuria, the renal origin of it showed low percentages. At any age, a blood work is always recommended in order to evaluate creatinine levels and renal function.

It is worth to remind that gross hematuria is a clinical scenario in which the clinician can make lots of mistakes. The first is to underestimate the relevance of hematuria once it has been resolved; it is important to note that in malignant pathology hematuria is normally intermittent, and this could explain the long time elapsed between the first symptom and the final diagnosis [5]. Another frequent mistake is to think that it is due to anticoagulant therapy. The authors results, as well as in others, this etiology is very infrequent, rather acts as a trigger in patients with an underlying cause $[4,10,12]$. Patients on anticoagulation therapy should not be excluded from investigations. Another frequent mistake is to attribute hematuria to a urine tract infection, some obsolete clinical guidelines 
suggest giving antibiotics to those patients who are diagnosed IGH; the results of this study and others suggest that this is not justified because such infections represent only $7.6 \%$ of the causes [14]. It is also a big mistake not to study patients who has already been diagnosed with a benign urological pathology which could have hematuria as a symptom, the most frequent of which is benign hypertrophy of the prostate. That is why it has been included in the study, unlike previously diagnosed malignant pathology. Given the high prevalence of BHP, a concomitant malignant cause is not excluded, just as one does not assume a hemorroidal pathology as a cause of rectorragia without ruling out malign pathology via colonoscopy.

A limitation of the study is that it is not a multicentric study with the corresponding population similarity. But it can be considered as a multicentric study, because it involves 400,000 inhabitants of different areas of Madrid in the population area, with a $20 \%$ population of immigrants, and with an enormous variety of population from all over the world. In this sense, studies from the USA Afro-Americans and Latin Americans have shown to have a greater predisposition to urological malignant pathology.

The authors think that one of the most relevant data is the $4.8 \%$ of mortality before diagnosis although it is certainly difficult to explain, but suggest the following possibilities: the patients age (mean age 70 years), the high comorbility, the fact that many of them had lung pathology due to their smoking habit and finally the delay of 95 days as an average before a final diagnosis is reached.

Some authors describe as criteria for hospitalization a hematocrit fall and/or the presence for AUR [15].

For all the above reasons, the authors suggest that gross hematuria should always be investigated and as soon as possible because it can be the first sign of a urological malignant pathology in many cases, and it can be a multidisciplinary task involving primary care physicians, emergency doctors, nephrologists and urologists [1].
It does not seem sensible then not to be aggresive in an early diagnosis. It is not justified, from any point of view including the economic cost, because diagnosis and treatment at an early stage, is six times cheaper than in patients with a more advanced disease [8]. Diagnostic protocols adapted to the characteristics of each institution should be drawn up in order to have a first valoration from the urologist within a matter of days after being seen in the Emergency department.

The most recent guidelines recommend the study of the lower urinary tract with an ultrasound and cystoscopy and the upper urinary tract with other diagnostic imaging study (tomography, ultrasound, etc.) although there is certain controversy because each imaging test has different sensitivity and specificity for each pathology; always including both, upper and lower tracts [1].

\section{References}

[1] G.D. Grossfeld, J.S.Jr. Wolf, M.S. Litwan, H. Hricak, C.L. Shuler, D.C. Agerter, et al., Asymptomatic microscopic hematuria in adults: summary of the AUA best practice policy recommendations, Am. Fam. Physician 63 (6) (2001) 1145-1154.

[2] J. Sutton, Evaluation of hematuria in adults, JAMA 263 (1990) 2475-2480.

[3] J.U. Juan Escudero, J.M. Esteban Hernández, M. Ramos de Campos, F. Ordoño Domínguez, M. Fabuel Deltoro, E. Marques Vidal, Hematuria de origen tumoral (Hematuria due to urothelial neoplasm), Actas. Urol. Esp. [Online] 32 (8) (2008), http://dx.doi.org/10.4321/ S0210-48062008000800018.

[4] R. Davis, J.S. Jones, D.A. Barocas, Diagnosis, Evaluation and Follow-up of Asymptomatic Microhematuria in Adults: AUA Guideline, May 2012.

[5] P.K. Rao, J.S. Jones, How to evaluate "dipstick hematuria”: what to do before you refer, Cleve. Clin. J. Med. 75 (3) (2008) 227-233.

[6] E.J. Yun, M.V. Meng, P.R. Carroll, Evaluation of the patient with hematuria, Med. Clin. North. Am. 88 (2) (2004) 329-343.

[7] R. Baeza, M. Cortés, M. Cáceres, G. Catalán, C. Flores, Hematuria macroscópica asintomática: diagnóstico etiológico, Mandujano Revista Chilena de Urología 84 (2004) 69.

[8] A.J. Mariani, M.C. Mariani, C. Macchioni, U.K. Stams, A. Hariharan, A. Moriera, The significance of adult 

Sign of Urologic Malignant Pathology

hematuria: 1.000 hematuria evaluations including a risk-benefit and cost-effectiveness analysis, J. Urol. 141 (1989) 350-355.

[9] R.J. Samblás García, M. De Cabo Ripill, J. Salinas Casado, Urgencias en Urología, Manual para Residentes, Hematuria, Jarpyo Editores, Madrid, 1995, pp. 96-108.

[10] T.R. Thaller, L.P. Wang. Evaluatión of asymptomatic microscopic hematuria in adults, Am. Fam. Physician 60 (4) (1999) 1143-1152, 1154.

[11] X. Shen, Diagnostic algorithm for the evaluation of hematuria, J. Am. Acad. Nurse. Pract. 22 (4) (2010) 186-191.

[12] J.G. Van Savage, F.A. Fried, Anticoagulant associated hematuria: a prospective study, J. Urol. 153 (5) (1995)
1594-1596.

[13] S.J. Foley, L.Z. Soloman, A.W. Wedderburn, K.M. Kashif, D. Summerton, V. Basketter, et al., A prospective study of natural history of hematuria associated with benign prostatic hyperplasia and the effect of 4 .finasteride, J. Urol. 163 (2) (2000) 496-498.

[14] C. Ritchie, E. Beran, Importance of occult hematuria found at screening, BMJ 292 (1986) 681.

[15] F. Sanchez-Carreras Aladrén, F. Leal Hernandez, I. Moncada Iribarren, E. Rodriguez Fernandez, J.M. Diez Cordero, Urgencias Urológicas: hematuria, in: Tema monográfico del LXI Cogreso Nacional de Urología 1996, pp. 73-83. 